\title{
Determination of chronic illness care and healthy lifestyles of patients hospitalized in internal medicine clinics
}

\section{Dahili kliniklerde yatan hastaların kronik hastalık bakımı ve sağlıklı yaşam biçimlerinin belirlenmesi}

\author{
Gülden ATAN $1 @$ (D) Gülcan BAHÇECİOĞLU TURAN ${ }^{2}$ (D) Zülfünaz ÖZER ${ }^{3}$ (D) \\ ${ }^{1}$ Faculty of Health Sciences, Van Yüzüncü Y1l University, 65090, Van-Turkey

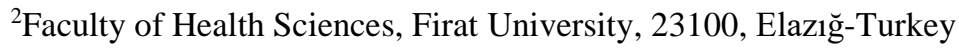 \\ ${ }^{3}$ Faculty of Health Sciences, Istanbul Sabahattin Zaim University, 34303, İstanbul-Turkey
}

Atıf gösterme/Cite this article as: Atan G, Bahçecioğlu Turan G, Özer Z. Determination of chronic illness care and healthy lifestyles of patients hospitalized in internal medicine clinics. ADYÜ Sağllk Bilimleri Derg. 2021;7(2):158-168. doi:10.30569.adiyamansaglik.887965

\begin{abstract}
Aim: This study aimed to determine the chronic disease care and healthy lifestyles of patients hospitalized in internal medicine clinics.

Materials and Methods: A descriptive and crosssectional study was conducted from May 1 to August 31, 2019. The study population consisted of 207 patients with chronic diseases.

Results: It was found that there was a statistically significant, and positively significant relationship was found between the chronic disease care assessment scale and the healthy lifestyle behaviors scale of the patients included in the study.

Conclusion: Positive association was detected between care satisfaction and healthy lifestyle behaviours of individuals with chronic disease. It may be recommended to provide training that supports the healthy lifestyles of these individuals who have to live with chronic diseases.
\end{abstract}

Keywords: Chronic illness; Healthy life; Internal clinic.

\begin{abstract}
Öz
Amaç: Bu çalışma, dahili kliniklerde yatan hastaların kronik hastalık bakımı ve sağlıklı yaşam biçimlerinin belirlenmesi amaciyla yapıldı.

Gereç ve Yöntem: Tanımlayıcı ve kesitsel olarak yapılan çalışma, Mayıs-Ağustos 2019 tarihleri arasında yürütülmüştür. Çalışmanın örneklemini ise kronik hastalığ́ olan 207 hasta oluşturdu.

Bulgular: Çalışmaya dahil edilen hastaların kronik hastalık bakımını değerlendirme ölçeği ile sağlıklı yaşam biçimi davranışları ölçeği arasında istatistiksel olarak önemli, pozitif yönde anlamlı bir ilişki olduğu saptand.

Sonuç: Kronik hastalığı olan bireylerin bakım memnuniyetleri ve sağlıklı yaşam biçimi davranışları arasında pozitif bir ilişki olduğu saptandı. Kronik hastalıkla yaşamak zorunda olan bu bireylerin sağlıklı yaşam biçimlerini destekleyici eğitimler verilmesi önerilmektedir.
\end{abstract}

Anahtar Kelimeler: Kronik hastalık; Sağlıklı yaşam; Dahili klinik.

Yazışma Adresi/Address for Correspondence: Gülden ATAN, Faculty of Health Sciences, Van Yüzüncü Yıl University, Department of Internal Medicine Nursing, 65090, Van-Turkey, E-mail: guldenatan@gmail.com

Geliş Tarihi/Received:28.02.2021 Kabul Tarihi/Accepted:24.06.2021 Yayım Tarihi/Published online:30.08.2021 


\section{Introduction}

Chronic diseases (CI) are those that involve a long life span, require continuous medical care and treatment, are slow and progressive, and cause irreversible changes in normal physiological functions. ${ }^{1}$ In western countries, chronic $\mathrm{CI}$ is rapidly growing and it is associated with increased number of days in the intensive care unit (ICU), prolonged hospitalization in post-acute(weaning) centers, and poor prognosis in the long term, which is also valid for our country. ${ }^{2}$

The factors causing increase in the incidence and prevalence of chronic illnesses are listed as the aging world population, stressors caused by rapid urbanization and decrease in physical activity with developing technology and changes in lifestyle such as changes in dietary habits. ${ }^{3}$

Although a great number of diseases such as cardiovascular diseases, diabetes, obesity, some cancers and chronic respiratory system diseases are among chronic illness group, most of them have common risk factors and prevention strategies. ${ }^{4}$ According to $\mathrm{WHO}$, health systems which are structured to provide mainly acute care services in chronic health problems are insufficient. More focus is placed on acute health problems than protective and preventive health services in the world and treatment services are mostly conducted through medication and technological interventions. ${ }^{5}$ Similarly, health system also focuses on treatment services in Turkey. Chronic diseases can be controlled significantly with programs targeting good health by focusing on protection measures and controlling risk factors. ${ }^{2}$ The most important characteristic of chronic illnesses is that a great number of underlying reasons except for some irreversible reasons such as family history and genetic background are completely preventable risk factors. Behavioural risk factors such as tobacco use, immobility and unhealthy diet are responsible for the occurrence of coronary heart disease and cerebrovascular diseases with a rate of $80 \% .^{3}$

Today, it is known that morbidity and mortality in chronic illnesses such as cardiovascular diseases, cancer, heart diseases, hypertension and diabetes can be decreased significantly with changes in lifestyle. ${ }^{6}$ In this context, it is important for individuals with chronic illness to adopt healthy lifestyle behaviours from the moment they are diagnosed. Healthy lifestyle behaviours are defined as behaviours which serve individuals to maintain and increase their levels of well-being. These behaviours include having a sufficient and balanced diet, not smoking, stress management, regular physical activity, effective spiritual development, positive interpersonal relationships and taking responsibility to maintain and develop health. ${ }^{7}$

Among the health team, nurses have important responsibilities such as creating awareness in society about the prevention of cardiovascular and other chronic illnesses, delaying the occurrence of the disease in risky individuals and decreasing possible complications and educating individuals, introducing them with healthy lifestyle habits and consulting them to adopt to treatment when they get ill. ${ }^{8}$

In the management of chronic illness, it is important to adopt healthy lifestyle behaviours, to control all behaviours that can influence the individual's health and to adopt daily activities according to the individual's health status. ${ }^{9}$ In this respect, it is thought that the present study would be a guide in planning and preparing a content for trainings and health practices to be conducted to inform individuals with chronic illness about the risk factors and the complications that may develop as a result of the illness and to prevent these complications. It is also thought that all these outcomes will contribute to the nursing care provided to individuals.

\section{Materials and Methods}

\section{Study design and sampling}

This descriptive and cross-sectional study was conducted to determine the chronic illness care and health lifestyles of patients hospitalized in internal medicine clinics.

\section{Population and sample of the study}


The population of the study consisted of 300 patients hospitalized in the internal clinics of a university hospital between May and August 2019.

The study sample consisted of 207 hospitalized patients with chronic diseases like respiratory system, diabetes, cardiovascular system treated in Internal Medicine Department of University from May 1 to August 31, 2019.

All patients were $\geq 18$ years old, and had no audial, visual and mental problems, volunteered to participate in the study and who had the cognitive competence to answer the questions.

17 patients in the hospital did not want to participate in the study, and 14 patients did not meet the research criteria. In the power analysis performed to determine the adequacy of the sample size, it was determined that the effect size was 2.25 (high level) and the power was 0.99 at the $95 \%$ confidence interval at the 0.05 significance level. These values show that the sample size is at the desired level.

\section{Data collection tools}

The data were collected by the researchers through face-to-face interview technique by using "Descriptive Information Form", "Patient Assessment of Chronic Illness Care" and "Healthy Lifestyle Behaviour Scale".

Descriptive information form: This form, consisting of 13 questions about the sociodemographic characteristics of individuals, was prepared using the relevant literature and studies.

Patient assessment of chronic illness care (PACIC): Turkish validity and reliability of the scale which was developed by Glasgow et al based on the Chronic Care Model of Wagner, was conducted by İncirkuş and Nahcivan. ${ }^{10,11}$ It is an easily applicable short instrument allowing patients to assess the health care services and at the same time which provides information about the quality of care services given. It is a Likert type scale with 20 items and it includes five subdimensions as patient activation (questions 13 ), decision support (questions 4-6), goal setting (questions 7-11); problem solving (questions 12-15) and follow-up/coordination (questions 16-20). The scale is scored as " $1=$ =never, $2=$ rarely, $3=$ =sometimes, $4=$ most of the time and 5=always". The total score of the scale is calculated from the average score of all 20 questions. Increased scale scores show that individual with chronic illness have high level of satisfaction from the care they receive and that chronic illness management is sufficient. ${ }^{11}$ According to the Turkish validity and reliability study of the scale; the Cronbach Alpha value is $0.91 .^{11}$

Healthy lifestyle behaviour scale (HLBS II): It was revised by Walker et al. and named HLBS II. ${ }^{12}$ Validity and reliability studies of the scale were conducted by Bahar et al. ${ }^{13}$ HLBS II is a 4-Likert type scale with 52 items and options of "never", "sometimes", "frequently" and "regularly". The scale consists of six sub-dimensions titled "health responsibility", "physical activity", "nutrition", "spiritual development", "interpersonal relations" and "stress management". The lowest score one can get from the scale is 52, while the highest score is 208. As the total score increases, it is accepted that the patient has healthier lifestyle behaviours. Chronbach Alpha value for HLBS II total scale is $0.92 .{ }^{13}$

\section{Assessment of data}

Descriptive statistics in the study were given as numbers, percentage, arithmetic mean and standard deviation. Independent Correlation Coefficient was used to assess the data obtained. SPSS 25 program was used for statistical analyses and level of significance was taken as 0,05 (p-value) in statistical analyses.

\section{Ethical principles of the study}

Approval was taken from the Ethical Board of the State University the study was conducted in (21/05/2019 date and 2019/0301 number) and written permission was taken from University. In addition, written and oral consent was taken from the individuals participating the study after the purpose of the study was explained. Written permission was obtained from the authors who conducted the Turkish validity and reliability of the study. 
The study was conducted in accordance with the Helsinki declaration principles.

\section{Results}

It was found that of the 207 individuals included in the study, $45.41 \%$ were female; $37.68 \%$ were illiterate; $69.57 \%$ were married and 84.06 were not working. It was also found that $36.71 \%$ were living in village and $44.93 \%$ had low level of income. Table 1 demonstrates demographic and chronic illness related information of the patients included in the study.

Table 1. Demographic information of patients included in the study.

\begin{tabular}{|c|c|c|}
\hline & & n $(\%)$ \\
\hline \multirow[t]{2}{*}{ Age } & $18-44$ years & $100(48.31)$ \\
\hline & $\geq 45$ years & $107(51.69)$ \\
\hline \multirow[t]{2}{*}{ Gender } & Female & $94(45.41)$ \\
\hline & Male & $113(54.59)$ \\
\hline \multirow[t]{4}{*}{ Level of education } & Illiterate & $78(37.68)$ \\
\hline & Primary & $78(37.68)$ \\
\hline & High School & $32(15.46)$ \\
\hline & Undergraduate and higher & $19(9.18)$ \\
\hline \multirow[t]{2}{*}{ Marital status } & Married & $144(69.57)$ \\
\hline & Single & $63(30.43)$ \\
\hline \multirow[t]{2}{*}{ Employment } & Employed & $33(15.94)$ \\
\hline & Unemployed & $174(84.06)$ \\
\hline \multirow[t]{2}{*}{ Social security } & Yes & $108(52.17)$ \\
\hline & No & $99(47.83)$ \\
\hline \multirow[t]{2}{*}{ Level of income } & Low & $93(44.93)$ \\
\hline & Moderate & $114(55.07)$ \\
\hline \multirow{3}{*}{ Place of residence } & Village & $76(36.71)$ \\
\hline & Town & $60(28.99)$ \\
\hline & City & $71(34.3)$ \\
\hline \multirow[t]{8}{*}{ Treatment unit } & $\begin{array}{l}\text { Internal medicine (DM, Cancer } \\
\text { GISS) }\end{array}$ & $76(36.71)$ \\
\hline & Cardiology & $18(8.7)$ \\
\hline & Neurology & $20(9.66)$ \\
\hline & Chest Diseases & $27(13.04)$ \\
\hline & Other units (Infectious Diseases, & $66(31.88)$ \\
\hline & Dermatology, & \\
\hline & Physical Therapy and Rehabilitation & \\
\hline & Center) & \\
\hline \multirow[t]{2}{*}{ Length of hospital stay } & 1-29 days & $170(82.13)$ \\
\hline & $\geq 30$ days & $37(17.87)$ \\
\hline \multirow[t]{5}{*}{ Chronic illness } & Respiratory system & $80(38.65)$ \\
\hline & Diabetes & $36(17.39)$ \\
\hline & Cardiovascular system & $41(19.81)$ \\
\hline & Cancer & $9(4.35)$ \\
\hline & GİS & $41(19.81)$ \\
\hline \multirow[t]{5}{*}{ Individual opinion about personal health } & Perfect & $4(1.93)$ \\
\hline & Very good & $7(3.38)$ \\
\hline & Good & $73(35.27)$ \\
\hline & Not bad & $86(41.55)$ \\
\hline & Bad & $37(17.87)$ \\
\hline \multirow[t]{3}{*}{ State of smoking } & Yes & $41(19.81)$ \\
\hline & No & $128(61.84)$ \\
\hline & Quit & $38(18.36)$ \\
\hline
\end{tabular}

In this study, the distribution of scores taken from PACIC was not given in any table; however, average score was found to be $2.94 \pm 0.49$. Average scores taken from patient activation sub-dimension was $3.32 \pm 0.84$; average score taken from decision support sub-dimension was $2.65 \pm 0.66$; average score taken from goal setting sub-dimension was $2.67 \pm 0.62$; average score taken from problem solving sub-dimension was $3.40 \pm 0.77$; while average score taken from follow-up and coordination sub-dimension was $2.78 \pm 0.56 \mathrm{In}$ 
this study, Cronbach Alpha value was calculated as 0.82 . (Table 2).

In the study, general total score from HLSBS-II was found to be $122.87 \pm 14.38$. Also, Cronbach Alpha value was calculated as 0.85.The total score taken from health responsibility sub-dimension was $22.14 \pm 3.49$; the total score taken from physical activity sub-dimension was $15.16 \pm 3.02$; the total score taken from nutrition sub-dimension was $20.24 \pm 3.07$; the total score taken from spiritual development sub-dimension was $24.31 \pm 3.96$; the total score taken from interpersonal relationships sub-dimension was $22.56 \pm 3.69$ and the total score taken from stress management sub-dimension was $18.45 \pm 3.56$ (Table 2).

Table 2. Mean scores of HLSBS-II *, PACIC ** total and sub-dimensions score.

\begin{tabular}{llll}
\hline HLSBS-II Scale & Mean \pm SD & PACIC Scale & Mean \pm SD \\
\hline Health responsibility & $22.14 \pm 3.49$ & Patient activation & $3.32 \pm 0.84$ \\
Physical activity & $15.16 \pm 3.02$ & Decision making support & $2.65 \pm 0.66$ \\
Nutrition & $20.24 \pm 3.07$ & Goal setting/ tailoring & $2.67 \pm 0.62$ \\
Spiritual development & $24.31 \pm 3.96$ & Problem solving & $3.40 \pm 0.77$ \\
$\begin{array}{l}\text { Interpersonal } \\
\text { relationships }\end{array}$ & $22.56 \pm 3.69$ & Follow-up/coordination & $2.78 \pm 0.56$ \\
$\begin{array}{l}\text { Stress management } \\
\text { Total }\end{array}$ & $18.45 \pm 3.56$. & & \\
\hline & $122.87 \pm 14.38$ & & $2.94 \pm 0.49$
\end{tabular}

Statistically significant difference was found the patients' PACIC total score averages of the patients who had an educational status of undergraduate and higher were found to be significantly higher when compared to those having "primary education" as educational status $(p=0.016)$. Patient activation score averages of patients having hospital stay periods of 1-29 days were significantly higher compared to those of patients who had $\geq 30$ days of hospital stay $(p=0.010)$. Goal setting/tailoring score averages of the patients in chest diseases, internal diseases and neurology units were significantly higher than those of the patients treated in other units $(p=0.016)$. Problem solving average scores of male patients were significantly higher when compared with those of female patients $(p=0.041)$. Similarly, it was concluded that problem solving average scores of the patients with an educational status of undergraduate and higher $(p=0.034)$. Follow-up/ coordination average scores of the patients with an educational status of undergraduate and higher were significantly higher when compared to those with an educational status of high school $(p=0.008)$ (Table 3$)$.
Statistically significant difference were found HLBS-II total score averages of the patients who were $\geq 45$ years old $(p=0.008)$, employed $(p=0.007)$, treated in cardiology units $(p=0.020)$, and those who assessed their general health condition as perfect $(p=0.024)$. Statistically significant difference was found health responsibility average scores of the patients between 18 and 44 years of age $(p=0.015)$. Spiritual development average scores of the patients who were employed were significantly higher than those who were not employed $(p=0.019)$. Interpersonal relationships average scores of the patients who were between 18 and 44 years of age were significantly higher than those who were 45 years and older $(p=0.030)$. In addition, interpersonal relationships average scores of patients who were high school graduates $(p=0.008)$ and who were employed $(p=0.005)$ were significantly higher. Stress management average scores of the patients who were employed were significantly higher than those of the patients who were unemployed $(p=0.004) \quad$ (Table 3). Similarly, stress management average scores of the patients who had moderate level of income $(p=0.040)$, who were treated in cardiology unit $(p=0.009)$ or those having a hospital stay between 1 
to 29 days $(p=0.017$ ) were significantly higher (Table 4$)$. There was no statistically significant relationship between other sociodemographic

Table 3. Comparison of PACIC total and sub-dimension scores in terms of the demographic characteristics of the patients included in the study.

\begin{tabular}{|c|c|c|c|c|c|c|c|}
\hline & & PACIC Total & Patient Activation & $\begin{array}{c}\text { Decision Making } \\
\text { Support }\end{array}$ & $\begin{array}{c}\text { Goal Setting/ } \\
\text { Tailoring }\end{array}$ & $\begin{array}{l}\text { Problem } \\
\text { Solving }\end{array}$ & $\begin{array}{c}\text { Follow-up/ } \\
\text { Coordination }\end{array}$ \\
\hline & 18-44 years & $2.97 \pm 0.42$ & $3.39 \pm 0.71$ & $2.7 \pm 0.66$ & $2.71 \pm 0.59$ & $3.44 \pm 0.78$ & $2.75 \pm 0.56$ \\
\hline \multirow[t]{3}{*}{ Age } & $\geq 45$ years & $2.91 \pm 0.54$ & $3.25 \pm 0.94$ & $2.61 \pm 0.66$ & $2.63 \pm 0.64$ & $3.36 \pm 0.76$ & $2.81 \pm 0.56$ \\
\hline & $p$ & 0.582 & 0.414 & 0.140 & 0.419 & 0.903 & 0.536 \\
\hline & Female & $2.9 \pm 0.49$ & $3.25 \pm 0.85$ & $2.66 \pm 0.65$ & $2.66 \pm 0.62$ & $3.3 \pm 0.8$ & $2.76 \pm 0.59$ \\
\hline \multirow{4}{*}{ Gender } & Male & $2.97 \pm 0.48$ & $3.38 \pm 0.82$ & $2.65 \pm 0.67$ & $2.68 \pm 0.62$ & $3.48 \pm 0.74$ & $2.79 \pm 0.54$ \\
\hline & $p$ & 0.270 & 0.407 & 0.424 & 0.995 & a 0.041 & 0.674 \\
\hline & Illiterate & $2.97 \pm 0.49$ & $3.37 \pm 0.86$ & $2.63 \pm 0.63$ & $2.64 \pm 0.62$ & $3.43 \pm 0.65$ & $2.91 \pm 0.51$ \\
\hline & Primary & $2.84 \pm 0.47$ & $3.23 \pm 0.82$ & $2.63 \pm 0.66$ & $2.6 \pm 0.6$ & $3.22 \pm 0.78$ & $2.66 \pm 0.58$ \\
\hline \multirow[t]{4}{*}{ Level of education } & High school & $2.96 \pm 0.51$ & $3.36 \pm 0.85$ & $2.67 \pm 0.67$ & $2.75 \pm 0.57$ & $3.55 \pm 0.92$ & $2.63 \pm 0.56$ \\
\hline & Undergraduate and higher & $3.15 \pm 0.41$ & $3.44 \pm 0.82$ & $2.81 \pm 0.81$ & $2.92 \pm 0.68$ & $3.71 \pm 0.79$ & $2.97 \pm 0.55$ \\
\hline & $p$ & ${ }^{b} 0.016$ & 0.560 & 0.952 & 0.295 & b 0.034 & b 0.008 \\
\hline & Married & $2.91 \pm 0.51$ & $3.26 \pm 0.91$ & $2.65 \pm 0.69$ & $2.66 \pm 0.63$ & $3.34 \pm 0.78$ & $2.77 \pm 0.59$ \\
\hline \multirow[t]{3}{*}{ Marital status } & Single & $3 \pm 0.41$ & $3.47 \pm 0.63$ & $2.66 \pm 0.58$ & $2.7 \pm 0.58$ & $3.54 \pm 0.72$ & $2.81 \pm 0.49$ \\
\hline & $p$ & 0.311 & 0.241 & 0.993 & 0.916 & 0.053 & 0.582 \\
\hline & Employed & $3.05 \pm 0.42$ & $3.52 \pm 0.69$ & $2.74 \pm 0.7$ & $2.79 \pm 0.65$ & $3.6 \pm 0.72$ & $2.78 \pm 0.56$ \\
\hline \multirow[t]{3}{*}{ Employment } & Unemployed & $2.92 \pm 0.49$ & $3.28 \pm 0.86$ & $2.64 \pm 0.65$ & $2.65 \pm 0.61$ & $3.36 \pm 0.77$ & $2.78 \pm 0.56$ \\
\hline & $p$ & 0.131 & 0.213 & 0.645 & 0.329 & 0.134 & 0.744 \\
\hline & Low & $2.88 \pm 0.47$ & $3.21 \pm 0.85$ & $2.61 \pm 0.58$ & $2.63 \pm 0.61$ & $3.31 \pm 0.74$ & $2.77 \pm 0.54$ \\
\hline \multirow[t]{4}{*}{ Level of income } & Moderate & $2.98 \pm 0.49$ & $3.41 \pm 0.82$ & $2.69 \pm 0.72$ & $2.7 \pm 0.62$ & $3.47 \pm 0.78$ & $2.78 \pm 0.58$ \\
\hline & $p$ & 0.370 & 0.169 & 0.967 & 0.669 & 0.230 & 0.806 \\
\hline & Internal medicine & $2.97 \pm 0.37$ & $3.37 \pm 0.72$ & $2.64 \pm 0.62$ & $2.74 \pm 0.53$ & $3.35 \pm 0.76$ & $2.86 \pm 0.44$ \\
\hline & Cardiology & $2.99 \pm 0.44$ & $3.35 \pm 0.8$ & $2.74 \pm 0.54$ & $2.64 \pm 0.68$ & $3.58 \pm 0.65$ & $2.78 \pm 0.54$ \\
\hline \multirow{4}{*}{ Treatment unit } & Neurology & $2.99 \pm 0.6$ & $3.47 \pm 0.95$ & $2.88 \pm 0.82$ & $2.85 \pm 0.85$ & $3.25 \pm 0.82$ & $2.69 \pm 0.69$ \\
\hline & Chest diseases & $2.91 \pm 0.49$ & $3.2 \pm 0.9$ & $2.62 \pm 0.71$ & $2.73 \pm 0.51$ & $3.31 \pm 0.72$ & $2.78 \pm 0.69$ \\
\hline & Other & $2.88 \pm 0.58$ & $3.25 \pm 0.92$ & $2.59 \pm 0.66$ & $2.52 \pm 0.63$ & $3.48 \pm 0.81$ & $2.71 \pm 0.6$ \\
\hline & $p$ & 0.820 & 0.597 & 0.558 & b 0.016 & 0.329 & 0.719 \\
\hline \multirow{3}{*}{$\begin{array}{l}\text { Length of hospital } \\
\text { stay }\end{array}$} & 1-29 days & $2.96 \pm 0.46$ & $3.39 \pm 0.8$ & $2.68 \pm 0.62$ & $2.69 \pm 0.6$ & $3.4 \pm 0.77$ & $2.78 \pm 0.55$ \\
\hline & $\geq 30$ days & $2.84 \pm 0.58$ & $2.97 \pm 0.94$ & $2.53 \pm 0.8$ & $2.56 \pm 0.69$ & $3.41 \pm 0.76$ & $2.76 \pm 0.63$ \\
\hline & $p$ & 0.480 & a 0.010 & 0.089 & 0.227 & 0.746 & 0.929 \\
\hline \multirow{6}{*}{ Chronic illness } & Respiratory system & $2.89 \pm 0.49$ & $3.24 \pm 0.86$ & $2.68 \pm 0.67$ & $2.69 \pm 0.62$ & $3.27 \pm 0.84$ & $2.71 \pm 0.59$ \\
\hline & Diabetes & $3.03 \pm 0.46$ & $3.41 \pm 0.82$ & $2.73 \pm 0.75$ & $2.69 \pm 0.62$ & $3.64 \pm 0.61$ & $2.83 \pm 0.58$ \\
\hline & Cardiovascular system & $2.95 \pm 0.48$ & $3.32 \pm 0.81$ & $2.63 \pm 0.66$ & $2.76 \pm 0.59$ & $3.44 \pm 0.75$ & $2.73 \pm 0.52$ \\
\hline & Cancer & $2.75 \pm 0.96$ & $3 \pm 1.4$ & $2.7 \pm 1.09$ & $2.47 \pm 1.12$ & $3 \pm 1.12$ & $2.71 \pm 0.79$ \\
\hline & GIS & $2.97 \pm 0.35$ & $3.46 \pm 0.68$ & $2.54 \pm 0.41$ & $2.57 \pm 0.47$ & $3.48 \pm 0.6$ & $2.93 \pm 0.45$ \\
\hline & $p$ & 0.651 & 0.583 & 0.670 & 0.495 & 0.111 & 0.211 \\
\hline
\end{tabular}


Descriptive statistics were given as average \pm standard deviation. a. $p<0.05$ and Mann-Whitney $\mathrm{U}$ test were used. b. $p<0.05$ and Kruskal-Wallis $\mathrm{H}$ test were used.

Table 4. Comparison of total score and sub-dimension scores of HLBS-II in patients included in the study in terms of demographic characteristics.

\begin{tabular}{|c|c|c|c|c|c|c|c|c|}
\hline & & HLBS Total & $\begin{array}{c}\text { Health } \\
\text { Responsibility }\end{array}$ & $\begin{array}{l}\text { Physical } \\
\text { Activity }\end{array}$ & Nutrition & $\begin{array}{c}\text { Spiritual } \\
\text { Development }\end{array}$ & $\begin{array}{l}\text { Interpersonal } \\
\text { Relationships }\end{array}$ & $\begin{array}{c}\text { Stress } \\
\text { Management }\end{array}$ \\
\hline & 18-44 years & $125.19 \pm 13.82$ & $22.8 \pm 3.07$ & $15.44 \pm 2.88$ & $20.38 \pm 2.92$ & $24.81 \pm 3.35$ & $23.13 \pm 3.44$ & $18.63 \pm 3.67$ \\
\hline \multirow[t]{3}{*}{ Age } & $\geq 45$ years & $120.7 \pm 14.62$ & $21.53 \pm 3.75$ & $14.91 \pm 3.14$ & $20.11 \pm 3.22$ & $23.84 \pm 4.43$ & $22.02 \pm 3.85$ & $18.29 \pm 3.47$ \\
\hline & $p$ & ${ }^{a} 0.008$ & a 0.015 & 0.205 & 0.436 & 0.076 & ${ }^{\mathbf{c}} \mathbf{0 . 0 3 0}$ & 0.374 \\
\hline & Female & $123.72 \pm 17.08$ & $22.47 \pm 3.73$ & $15.47 \pm 3.21$ & $20.31 \pm 3.21$ & $24.37 \pm 4.47$ & $22.34 \pm 3.96$ & $18.77 \pm 4.09$ \\
\hline \multirow[t]{3}{*}{ Gender } & Male & $122.16 \pm 11.71$ & $21.88 \pm 3.27$ & $14.91 \pm 2.85$ & $20.19 \pm 2.97$ & $24.26 \pm 3.5$ & $22.73 \pm 3.46$ & $18.19 \pm 3.05$ \\
\hline & $p$ & 0.439 & 0.248 & 0.187 & 0.934 & 0.838 & 0.446 & 0.647 \\
\hline & Illiterate & $121.03 \pm 15.93$ & $21.5 \pm 3.82$ & $15.13 \pm 3.18$ & $20.26 \pm 3.28$ & $24.13 \pm 4.5$ & $21.67 \pm 3.87$ & $18.35 \pm 3.6$ \\
\hline \multirow{5}{*}{$\begin{array}{l}\text { Level of } \\
\text { education }\end{array}$} & Primary & $121.69 \pm 12.97$ & $21.99 \pm 3.42$ & $14.96 \pm 2.95$ & $19.94 \pm 2.99$ & $24 \pm 3.75$ & $22.59 \pm 3.3$ & $18.22 \pm 3.52$ \\
\hline & High school & $127.34 \pm 12.95$ & $23.59 \pm 2.73$ & $15 \pm 2.9$ & $20.63 \pm 2.43$ & $24.97 \pm 3.42$ & $24.22 \pm 3.26$ & $18.94 \pm 3.73$ \\
\hline & Undergraduate and higher & $127.74 \pm 13.87$ & $23 \pm 2.73$ & $16.42 \pm 2.76$ & $20.79 \pm 3.54$ & $25.21 \pm 3.26$ & $23.26 \pm 4.23$ & $19.05 \pm 3.41$ \\
\hline & $p$ & ${ }^{b} \mathbf{0 . 0 2 8}$ & 0.077 & 0.291 & 0.472 & 0.477 & ${ }^{d} 0.008$ & 0.472 \\
\hline & Married & $123.76 \pm 16$ & $22.01 \pm 3.9$ & $15.3 \pm 3.32$ & $20.51 \pm 3.41$ & $24.31 \pm 4.26$ & $22.82 \pm 3.81$ & $18.81 \pm 3.85$ \\
\hline \multirow[t]{2}{*}{ Marital status } & Single & $120.84 \pm 9.52$ & $22.46 \pm 2.28$ & $14.86 \pm 2.18$ & $19.62 \pm 2.02$ & $24.3 \pm 3.22$ & $21.95 \pm 3.36$ & $17.65 \pm 2.66$ \\
\hline & $p$ & 0.679 & 0.653 & 0.259 & 0.166 & 0.984 & 0.120 & 0.086 \\
\hline \multirow{4}{*}{$\begin{array}{l}\text { Employment } \\
\text { status }\end{array}$} & Employed & $129.55 \pm 13.96$ & $23.42 \pm 2.85$ & $15.67 \pm 3.54$ & $20.45 \pm 3.26$ & $25.79 \pm 3.04$ & $24.21 \pm 3.24$ & $20 \pm 3.87$ \\
\hline & Unemployed & $121.6 \pm 14.15$ & $21.9 \pm 3.55$ & $15.07 \pm 2.91$ & $20.2 \pm 3.04$ & $24.03 \pm 4.06$ & $22.24 \pm 3.7$ & $18.16 \pm 3.43$ \\
\hline & $p$ & a 0.007 & 0.083 & 0.298 & 0.988 & ${ }^{c} 0.019$ & c 0.005 & a 0.004 \\
\hline & Low & $121.49 \pm 14.16$ & $21.66 \pm 3.44$ & $15.02 \pm 2.74$ & $20.24 \pm 3.08$ & $24.02 \pm 4.36$ & $22.49 \pm 3.6$ & $18.06 \pm 3.6$ \\
\hline \multirow[t]{4}{*}{ Level of income } & Moderate & $123.99 \pm 14.52$ & $22.54 \pm 3.48$ & $15.28 \pm 3.24$ & $20.25 \pm 3.08$ & $24.54 \pm 3.61$ & $22.61 \pm 3.78$ & $18.77 \pm 3.51$ \\
\hline & $p$ & 0.177 & 0.076 & 0.540 & 0.854 & 0.356 & 0.831 & a 0.040 \\
\hline & Internal medicine & $125.93 \pm 15.27$ & $22.38 \pm 3.32$ & $15.74 \pm 2.99$ & $20.84 \pm 3.31$ & $24.88 \pm 3.87$ & $23.01 \pm 3.38$ & $19.08 \pm 3.76$ \\
\hline & Cardiology & $126.39 \pm 12.3$ & $24.17 \pm 3.09$ & $15.56 \pm 2.75$ & $19.78 \pm 2.05$ & $24.89 \pm 4.01$ & $22.72 \pm 2.95$ & $19.28 \pm 3.18$ \\
\hline \multirow{4}{*}{ Unit of treatment } & Neurology & $124.25 \pm 14.26$ & $22.05 \pm 3.27$ & $15.3 \pm 3.44$ & $20.8 \pm 3.38$ & $24.2 \pm 4.73$ & $22.75 \pm 3.67$ & $19.15 \pm 4.69$ \\
\hline & Chest diseases & $116.67 \pm 15.14$ & $20.89 \pm 3.38$ & $14.22 \pm 3.65$ & $20.15 \pm 2.4$ & $23.07 \pm 4.11$ & $21.26 \pm 4.38$ & $17.07 \pm 3.02$ \\
\hline & Other & $120.5 \pm 12.62$ & $21.86 \pm 3.7$ & $14.74 \pm 2.62$ & $19.55 \pm 3.07$ & $24.03 \pm 3.71$ & $22.45 \pm 3.9$ & $17.86 \pm 3.03$ \\
\hline & $p$ & ${ }^{b} 0.020$ & b 0.020 & 0.135 & 0.102 & 0.295 & 0.328 & b 0.009 \\
\hline \multirow{3}{*}{$\begin{array}{l}\text { Length of } \\
\text { hospital stay }\end{array}$} & 1-29 days & $124.01 \pm 14.58$ & $22.48 \pm 3.4$ & $15.28 \pm 2.99$ & $20.25 \pm 3$ & $24.54 \pm 4.04$ & $22.75 \pm 3.72$ & $18.72 \pm 3.73$ \\
\hline & $\geq 30$ days & $117.65 \pm 12.3$ & $20.62 \pm 3.51$ & $14.65 \pm 3.13$ & $20.22 \pm 3.42$ & $23.27 \pm 3.45$ & $21.68 \pm 3.47$ & $17.22 \pm 2.3$ \\
\hline & $p$ & ${ }^{a} 0.009$ & a 0.003 & 0.253 & 0.998 & 0.078 & 0.110 & a 0.017 \\
\hline \multirow{6}{*}{ Chronic illness } & Respiratory system & $123.03 \pm 14.95$ & $22.05 \pm 3.45$ & $14.98 \pm 3.15$ & $20.63 \pm 3.03$ & $24.23 \pm 4.03$ & $22.53 \pm 3.89$ & $18.63 \pm 3.76$ \\
\hline & Diabetes & $123.25 \pm 13.27$ & $22.33 \pm 3.25$ & $14.64 \pm 3.13$ & $20.47 \pm 3.58$ & $24.28 \pm 4.05$ & $22.92 \pm 3.83$ & $18.61 \pm 3.7$ \\
\hline & Cardiovascular system & $126.15 \pm 14.76$ & $22.71 \pm 3.32$ & $15.9 \pm 2.98$ & $20.2 \pm 2.78$ & $25.29 \pm 3.99$ & $23.29 \pm 3.92$ & $18.76 \pm 3.86$ \\
\hline & Cancer & $108.56 \pm 13.7$ & $17.78 \pm 5.74$ & $14.67 \pm 4$ & $18 \pm 2.18$ & $20.67 \pm 4.44$ & $20.78 \pm 1.86$ & $16.67 \pm 2$ \\
\hline & GIS & $122.1 \pm 12.51$ & $22.56 \pm 2.72$ & $15.37 \pm 2.4$ & $19.83 \pm 2.99$ & $24.32 \pm 3.25$ & $21.95 \pm 3.1$ & $18.07 \pm 2.95$ \\
\hline & $p$ & 0.065 & 0.079 & 0.279 & 0.085 & 0.099 & 0.176 & 0.643 \\
\hline
\end{tabular}




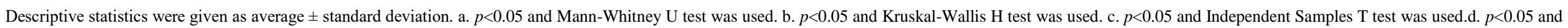
One-Way ANOVA test was used.

As a conclusion, a statistically significant, linear and weak association was found between chronic patient care assessment scale total scores and the scores of patient activation, decision making support, goal setting/tailoring, problem solving an follow-up coordination sub-dimensions. Also a statistically significant, linear and weak association was found between healthy lifestyle behaviours scale general total scores and the scores of health responsibility, physical activity, nutrition, spiritual development and stress management subdimensions (Table 5).

Table 5. Correlation between scale in general and sub-dimensions of the scale.

\begin{tabular}{|c|c|c|c|c|c|c|}
\hline & $\begin{array}{c}\text { PACIC } \\
\text { Total }\end{array}$ & Patient activation & $\begin{array}{c}\text { Decision making } \\
\text { support }\end{array}$ & Goal setting/tailoring & $\begin{array}{c}\text { Problem } \\
\text { solving }\end{array}$ & Follow-up/ Coordination \\
\hline HLSBS-II Total & $0.282 *$ & 0.238* & $0.182 *$ & $0.370 *$ & 0.118 & 0.050 \\
\hline Health responsibility & $0.269 *$ & $0.266^{*}$ & 0.126 & $0.247 *$ & $0.217 *$ & 0.118 \\
\hline Physical activity & $0.160 *$ & 0.122 & -0.107 & $0.185^{*}$ & 0.085 & $0.188 *$ \\
\hline Nutrition & $0.165 *$ & 0.048 & $0.163 *$ & $0.326 *$ & -0.036 & 0.048 \\
\hline Spiritual development & $0.309 *$ & $0.273 *$ & $0.141 *$ & $0.202 *$ & $0.241 *$ & 0.122 \\
\hline $\begin{array}{l}\text { Interpersonal } \\
\text { relationships }\end{array}$ & 0.110 & 0.113 & $0.236 *$ & $0.212 *$ & -0.003 & -0.084 \\
\hline Stress management & 0.137* & 0.114 & $0.169 *$ & $0.287 *$ & -0.039 & -0.070 \\
\hline
\end{tabular}

* $p<0.05$ and Spearman's correlation coefficient were used.

\section{Discussion}

In our study evaluating a mixed patient group, PACIC score average was found $2.94 \pm 0.49$. This result shows that patients included in the study had moderate level of care satisfaction and chronic illness management. When studies performed in similar patient groups were reviewed, average PACIC score was found between 2.44 and 3.17. The total average PACIC score in our study was similar to many studies in the literature, except other reports showing lower scores. ${ }^{14-16}$

It was determined that the highest score patients received from PACIC sub-dimensions was in "problem-solving" sub-dimension $(3.40 \pm 0.77)$, while the lowest score was in "decision making support" sub-dimension (2.65 \pm 0.66$)$. Unlike the results of our study, it was found in many studies that the patients got the highest score in "decision making support" sub-dimension; while the lowest score was in "follow-up/coordination" sub-dimension ${ }^{17-20}$ This result shows that patients included in the study should be supported in terms of deciding for their self-care.

When the patients' PACIC total and sub-dimension score averages were examined in terms of their level of education, it was found that total score of the scale and the sub-dimensions of problem solving and follow-up and coordination influenced level of education significantly and average scores increased as level of education increased. Similar results were found in studies in literature analysing mixed or specific patient groups. ${ }^{15,20,21}$ This can be related with the fact that educated individuals are better in receiving the necessary care, adaptation and self-management. 
In our study, it was found that gender affected only the sub-dimension of problem solving significantly and it was found to be higher in males $(p<0.05)$. In a study conducted with a mixed patient group. It found that gender influenced only the subdimension of goal setting significantly and that it was higher in males. ${ }^{2}$ In a study conducted on Type 2 diabetes patients, found that gender influenced total scale score and it was found to be higher in males. ${ }^{22}$ In some studies conducted, it was found that gender did not have any significant influence on PACIC total and sub-dimension score averages. $^{23-31}$

In our study, HLBS II total score average was found as $122.87 \pm 14.38$. In a study performed on patients with diabetes, found HLBS II total score average as $127.98 \pm 18.91 .^{7}$ But in another study found HLBS II total score average as $127.45 \pm 20.51$ in cardiac patients. ${ }^{24}$ Considering that the highest score one can get from HLBS II is 208 , it may be suggested that the patients in the present study have moderately healthy lifestyle behaviours.

When the sub-dimensions of HLBS II were examined, it was found that the highest score averages were in spiritual development $(24.31 \pm 3.36)$ and interpersonal relationships (22.56 \pm 3.69$)$, followed by health responsibility (22.14 \pm 3.49$)$, nutritional habits $(20.24 \pm 3.07)$ and stress management (18.45 \pm 3.56$)$. On the other hand, the lowest score average was found in physical activity sub-dimension (15.16 \pm 3.02$)$. In literature, sub-dimension score averages from the highest to the lowest are similar to our study results. ${ }^{9,25,26}$ In recent years, exercise has been recognized as an important intervention tool in preventing and rehabilitating chronic diseases. However, patients lead an inactive life due to the physical problems they experience. ${ }^{27}$ These results present that the habit of doing exercise is low in our patients.

In our study, when focusing on the patients' ages and their HLBS II score averages, it was found that as age increased, interpersonal relationships, health responsibility and HLBS II total scores decreased. In a study performed in cardiac patients, found that physical activity score decreased as age increased. ${ }^{24}$ But while another study detected that health responsibility, interpersonal relationships, physical activity, nutrition and HLBS II decreased with age in a study including nurses working in a surgical clinic. ${ }^{28}$ This result may be due to functional impairments that occur with increasing age, as well as decreased life satisfaction due to concomitant chronic diseases and thus a decrease in quality of life.

Unlike our study, it was found that age did not influence HLBS II total score and subdimension score averages in a study conducted on coronary artery patients. ${ }^{25}$ Similarly a study reported on healthy individuals. ${ }^{8}$ This difference might be due to the mixed patient group assessed in our study. When the association between patients' level of education and their HLBS II score averages was evaluated, it was found that HLBS II total score average and interpersonal relationships score average increased as the level of education increased. In a study they conducted on colorectal cancer patients, found that as the level of education increased, patients' spiritual development increased. ${ }^{29}$ In a study was conducted on cardiac patients, found that as the level of education increased. $^{4}$ Patients' healthy life style behaviours and quality of life increased. Our findings are parallel to the results of the studies in the literature. Therefore, it might be suggested that there is a directly proportional relationship between the level of education and teaching healthy lifestyle behaviours.

In our study, HLBS II total score, and subdimensions of spiritual development, interpersonal relationship and stress management were found to be higher in patients working when compared with those who were not working. In a study was detected HLBS II total score and subdimensions of spiritual development and physical activity to be higher in their study with heart patients. ${ }^{24}$ It is an expected result that disease metabolic states and thus physical functional abilities of patients who are working are better since working environment provides spiritual development. ${ }^{30}$ In addition, it was found in our study that the patients with 
social security had significantly higher health responsibility when compared with those who did not have any social security. Similarly, it was found in another study that HLBS total score average and averages of sub-dimensions of health responsibility, physical activity, spiritual development and interpersonal relationships were significantly higher in women who migrated and who had social security $(p<0.05) .{ }^{31}$ Similarly, it can be said that it is an expected situation for patients with social security to have high healthy lifestyle behaviours.

In our study, a significant association was found between HLBS II score averages in terms of the length of hospital stay and it was found that the patients whose length of hospital stay was 1-29 days had higher HLBS II total score and sub-dimension scores of health responsibility and stress management than those whose length of hospital stay was 30 days and longer. The results of the study show that individuals who are hospitalized for a long time do not care about healthy lifestyle behaviour.

Statistically significant and positive association was found between the chronic illness care assessment scale total score and patient activation, decision support, goal setting/tailoring, problem solving and followup/coordination scores of the patients included in the study and their healthy lifestyle behaviours scale total scores and health responsibility, physical activity, nutrition, spiritual development and stress management scores. In line with these findings, it can be thought that as care satisfaction of individuals with chronic illness and illness management increases, these will also cause positive changes in their healthy lifestyle behaviour.

\section{Limitations of the study}

This study is limited to patients hospitalized in internal medicine clinics of a single centre. The sample in this study reflects only one area of Turkey.

\section{Conclusion}

It was found that patients had moderate level of chronic illness care satisfaction. In addition, it was found that the highest score in the study was from problem solving subdimension, while the lowest score was detected in decision making support subdimension. It was found that patients had moderate level of healthy lifestyle behaviours and the highest score was in spiritual development sub-dimension, while the lowest score was in physical activity sub-dimension. In addition, it was determined in both scales that care satisfaction and healthy lifestyle behaviours increased when educational status increased and length of hospital stay was 1-29 days. Positive association was detected between care satisfaction and healthy lifestyle behaviours of individuals with chronic disease. In parallel with all these results, it may be suggested that the study be conducted with a larger sample group. In this case, it will be possible to provide a healthy review of the care given to the patients with chronic diseases and their results. In addition, it may be recommended to provide training that supports the healthy lifestyles of these individuals who have to live with chronic diseases.

\section{Ethics Committee Approval}

Approval was taken from the Ethical Board of the State University the study was conducted in (21/05/2019 date and 2019/0301 number) and written permission was taken from University. The study was conducted in accordance with the Helsinki declaration principles.

\section{Informed Consent}

All participants signed the Informed Consent Form and their consent was obtained.

\section{Author Contributions}

Idea, design, collection of resources, analysis and interpretation of results and literature, written and critical: GA, GBT and ZÖ.

\section{Acknowledgements}

We thank all the patients who agreed to participate in the research for their sincere sharing.

\section{Conflict of Interest}

There is no conflict of interest to declare. 


\section{Financial Disclosure}

There is no person/organization supporting this study financially.

\section{Peer-review}

\section{Externally peer-reviewed.}

\section{References}

1. Bektaş Akpınar N, Aşkın Ceran M, Karatay Üniversitesi Sağlık Bilimleri Yüksekokulu Hemşirelik Bölümü K, et al. Kronik Hastalıklar ve Rehabilitasyon Hemşireliği Chronic Diseases and Rehabilitation Nursing.2019; 3: 40-152.

2. Chiaranai C, Chularee S, Srithongluang S. Older people living with chronic illness. Geriatr Nurs (Minneap) 2018; 39: 513520.

3. Usuba K, Li AKC, Nowrouzi-Kia B. Trend of the burden of chronic illnesses: using the Canadian Community Health Survey. Public Health 2019; 177: 10-18.

4. Tshomo Y, Chaimongkol N. Prevalence of depression and its associated factors among persons with chronic medical illness in Bhutan. Arch Psychiatr Nurs 2019; 33: 347-351.

5. Karabulutlu EY, Atman R, Yağcı S. Evaluation care of persons with chronic illness. 2015;3:16-30.

6. Köse E, Güçiz Doğan B, Çetin Ekerbiçer H, et al. Study of Healthy Life Behaviors and Related Factors In Students In Sakarya University Medical Faculty Classes of I, II, III.Sakarya Tip Dergisi.2018;8:20-29.

7. Taskın Yılmaz F, Karakoc Kumsar A, Celik S. The Association Between Healthy Lifestyle Behaviors and Level of Knowledge About Cardiovascular Disease Risk Factors in People With Type 2 Diabetes. J Educ Res Nurs. Epub ahead of print 2018. DOI: 10.5222/head.2018.063.

8. Trindade IA, Barbosa R, Ferreira C, et al. Further validation of the cognitive fusion questionnaire - chronic illness (CFQ-CI) in different health condition samples. J Context Behav Sci 2020; 16: 45-48.

9. Çalışkan C, Arberk K, Üner S. Healthy lifestyle behaviours of university students. Türkiye Halk Sağglı̆̆ Derg 2018; 16: 2042013.

10. Glasgow RE, Wagner EH, Schaefer J, et al. Development and validation of the Patient Assessment of Chronic Illness Care (PACIC). Med Care 2005; 43: 436-444.

11. İncirkuş K, Nahcivan NÖ. Validity and Reliability Study of Turkish Version of The Patient Assessment of Chronic Illness Care-Patient Form. 2011;4:102-109.

12. Walker SN, Hill-Polerecky DM. Psychometric Evaluation of the Health-Promoting Lifestyle Profile II. Univ Nebraska Med Cent Omaha 1996; Unpublished.

13. Bahar Z, Beşer A, Gördes N, et al. Healthy Life Style Behavior Scale II:A Reliability And Validity Study. CÜ Hemsirelik Yüksekokulu Dergisi 2008; 12:1-13.

14. Schmittdiel J, Mosen DM, Glasgow RE, et al. Patient Assessment of Chronic Illness Care (PACIC) and improved patient-centered outcomes for chronic conditions. J Gen Intern Med 2008; 23: 77-80.

15. Taggart J, Chan B, Jayasinghe UW, et al. Patients Assessment of Chronic Illness Care (PACIC) in two Australian studies: Structure and utility. J Eval Clin Pract 2011; 17: 215-221.

16. Vrijhoef HJM, Berbee R, Wagner EH, et al. Quality of integrated chronic care measured by patient survey: Identification, selection and application of most appropriate instruments. Heal Expect 2009; 12: 417-429.

17. Cramm JM, Nieboer AP. Factorial validation of the patient assessment of chronic illness care (PACIC) and PACIC short version (PACIC-S) among cardiovascular disease patients in the Netherlands. Health Qual Life Outcomes 2012; 10: 104.

18. Jackson GL, Weinberger M, Hamilton NS, et al. Racial/ethnic and educational-level differences in diabetes care experiences in primary care. Prim Care Diabetes 2008; 2: 39-44.

19. Ludt S, Van Lieshout J, Campbell SM, et al. Identifying factors associated with experiences of coronary heart disease patients receiving structured chronic care and counselling in European primary care. BMC Health Serv Res 2012; 12: 221

20. Rosemann T, Laux G, Szecsenyi J, et al. The Chronic Care
Model: Congruency and predictors among primary care patients with osteoarthritis. Qual Saf Heal Care 2008; 17: 442-446.

21. Houle J, Beaulieu MD, Lussier MT, et al. Patients' experience of chronic illness care in a network of teaching settings. Can Fam Physician 2012; 58: 1366-1373.

22. Simonsen N, Koponen AM, Suominen S. Patients' assessment of chronic illness care: A validation study among patients with type 2 diabetes in Finland. BMC Health Serv Res; 18. Epub ahead of print June 2018. DOI: 10.1186/s12913-018-3206-7.

23. Glasgow RE, Whitesides $\mathrm{H}$, Nelson $\mathrm{CC}$, et al. Use of the patient assessment of chronic illness care (PACIC) with diabetic patients: Relationship to patient characteristics, receipt of care, and self-management. Diabetes Care 2005; 28: 2655 2661

24. Teuteberg D, Newcomb P, Sosa S. Nurse Practitioner Management of Uninsured, Rural Adults With Chronic Illness. J Nurse Pract 2019; 15: e77-e79.

25. Aksoy T, Uçar H.. Hemşirelik Öğrencilerinin Sağlıklı Yaşam Biçimi Davranısları. Hacettepe Üniversitesi Hemșirelik Fakültesi Dergisi 2014; 1(2): 53-67.

26. Kolaç N, Balcı AS, Şişman FN, Ataçer BE, Dinçer S. Fabrika çalışanlarında sağlıklı yaşam biçimi davranışı ve sağlık algısı. Bakırköy Tip Dergisi 2018; 14: 267-274.

27. Gould DW, Graham-Brown M, Watson EL, Viana JL, Smith AC.( 2014) Physiological benefits of exercise in pre-dialysis chronic disease. Nephrology 2014; 19(9): 519-527.

28. Yüceler Kaçmaz H, Çürük GN. Healthy lifestyle behaviours and attitudes of relatives of patients with colorectal cancer towards protection from colorectal cancer. Turkish J Clin Lab 2017; 9: 36-49.

29. Johansson P, Dahlström U, Broström A. Factors and interventions influencing health-related quality of life in patients with heart failure: A review of the literature. European Journal of Cardiovascular Nursing 2006; 5: 5-15.

30. Demir G, Arı̈z A. Healthy Life Style Behaviors of Migrant Women and Influencing Factors. Journal of Duzce University Health Sciences Institute 2014;4:1-8.

31. O'Connor SR, Connaghan J, Maguire R, et al. Healthcare professional perceived barriers and facilitators to discussing sexual wellbeing with patients after diagnosis of chronic illness: A mixed-methods evidence synthesis. Patient Education and Counseling 2019; 102: 850-863. 